\title{
Design and modeling of a photonic integrated device for optical vortex generation in a silicon waveguide
}

\author{
R.V. Kutluyarov ${ }^{1}$, D.M. Fatkhiev ${ }^{1}$, I.V.Stepanov ${ }^{1}$, E.P. Grakhova ${ }^{1}$, V.S. Lyubopytov ${ }^{2,1}$, A.Kh. Sultanov ${ }^{1}$ \\ ${ }^{1}$ Ufa State Aviation Technical University, 450008, Ufa, Russia, Karla Marxa Street 12, \\ ${ }^{2}$ Skolkovo Institute of Science and Technology, 121205, Moscow, Russia, Bolshoy Boulevard 30, bld. 1
}

\begin{abstract}
We propose and numerically verify a design of the photonic integrated circuit for in-plane generation of a $1^{\text {st }}$ azimuthal order vortex mode in dielectric rectangular waveguides. Radiation is introduced into the proposed structure in a standard way through two grating couplers. Applying a mode coupling and specific phase shift, a field with the required amplitude-phase distribution is formed directly in the output waveguide. The geometric dimensions of the device are simulated and optimized to fit the technological parameters of the silicon-on-insulator platform.

Keywords: orbital angular momentum, integrated photonics, optical waveguides, in-plane generation, silicon-on-insulator.

Citation: Kutluyarov RV, Fatkhiev DM, Stepanov IV, Grakhova EP, Lyubopytov VS, Sultanov AK. Design and modeling of a photonic integrated device for optical vortex generation in a silicon waveguide. Computer Optics 2021; 45(3): 324-330. DOI: 10.18287/2412-6179-CO-850.

Acknowledgments: This work was partially funded under the Grant of the Russian Science Foundation Project No. 19-49-04112 (simulations and optimization of the device parameters), and under the State Assignment from the Ministry of Science and Higher Education of the Russian Federation for Ufa State Aviation Technical University FEUE-2020-0007 (concept of the device and operation principle). Authors thank Computer Science Research Institute of the Ufa State Aviation Technical University for technical support of performed numerical computations on HPC cluster [https://www.ugatu.su/supercomputer/].
\end{abstract}

\section{Introduction}

In recent years, optical beams carrying orbital angular momentum (OAM), so-called optical vortices, have attracted significant attention from researchers due to their specific properties. Such beams are characterized by a helical wavefront and a phase proportional to $\exp (i l \phi)$, where $l$ is an integer known as the topological charge, which can take any value. Thus, optical OAM modes compose an orthogonal basis that can be applied for fundamental improvement of the capacity for fiber optical [1], free-space [2, 3], and quantum communications [4, 5]. Besides, the phase of optical vortices is used for the polarizing properties analysis of laser fields. Features of sharp focusing of Gaussian-Laguerre vortex laser beams for various types of uniform polarization depending on the order of the vortex phase is considered in [6]. The detection of the polarization state, as well as the relationship between phase and polarization features in optical systems with a high numerical aperture, are presented in [7, 8]. Finally, [9] demonstrates the focusing of displaced optical vortices of arbitrary order with different polarizations, and their propagation from the point of view of catastrophe theory is considered in [10].

To date, various applications have been proposed for optical vortices including optical tweezers and spanners [11-15], non-diverging speckles [16], imaging and microscopy [17-20], novel sensing technologies for detecting molecules and nanostructures [21-23], object motion detection $[24,25]$. Consequently, the demand for efficient methods of vortex beams generation increases.
Most methods for generating optical vortices are performed by discrete optical elements and are conventionally divided into two groups: spatial and fiber generating methods [26]. The devices from the first group include diffractive optical elements [27], cylindrical lens mode converters [28], spiral phase plates (SPPs) [29, 30], spatial light modulators [31], metamaterials [32] and q-plates [33]. The second group of fiber-based devices like fiber grating [34], chirally coupled core fiber [35] or photonic lanterns [36] seems to provide more promising solutions in terms of system size yet on the millimeter scale. However, in recent years, solutions based on photonic integrated circuits (PICs) have attracted the greatest interest due to their energy efficiency, small size, and performance stability. Besides, the available fabrication processes of PIC ensure the repeatability in the massive production of the developed devices. The OAM modes generator based on PIC satisfies completely the modern trend towards optical integration both in terms of functional miniaturization and in terms of multiplexing optical signals for transmission in a single propagation medium.

Existing PIC solutions for generation and (de)multiplexing of OAM-beams can be divided into two groups: out-of-plane and in-plane solutions. The out-ofplane vortex generation can be implemented by adding an integratable vortex generator element at the output port of the light source or generating a vortex beam inside the light source cavities directly [37]. Among the proposed designs there are solutions based on microring resonators with angular gratings [38-40], free-form metasurfaces, which can overcome the microring's bandwidth limita- 
tion [41], single-mode vertical-cavity surface-emitting laser (VCSEL) or tunable MEMS-based Fabry-Perot filter with the dielectric micro-sized SPPs deposited on its top $[42,43]$. Most methods for out-of-plane generation demonstrate satisfactory mode purity of the output OAM beam, however, its free-space propagation requires a lens to focus the signal and couple it into the fiber, which negatively affects the reliability and size of the final device. Another disadvantage of this approach is the limited potential for multiplexing of optical vortices with different topological charges.

Regarding the in-plane on-chip solutions, the generation and propagation of optical vortices are performed inside the dielectric waveguides, bringing the advantageous possibility to implement a vortex generator as part of more complex integrated devices. Obviously, PICs based on commercial fabrication processes, which are meant for single-mode applications are not suitable for vortex propagation within an integrated waveguide. However, today multi-project wafer (MPW) technologies customized for the fabrication of waveguides with an increased height that can be optimized for vortex beams propagation are available [44].

To date, several approaches to in-plane vortex generation have been proposed. In [45] a rectangular waveguide based on Silicon-On-Insulator (SOI) platform with a single longitudinal trench is used to split the mode degeneracy and excite two orthogonal LP-like eigenmodes with different propagation constants for further synthesis of OAM modes with topological charges $l=+1$ or $l=-1$. Another idea is to use hybrid plasmonic waveguides, e.g. in [46] the copper layer is located on top of the silicon nanowire with silica spacer between them; in [47] Lshaped asymmetric copper strip is located on the right top of the square silicon core; [48] proposes applying graphene. It should be noted that in the last three works, the OAM beam is formed on the longitudinal component of the electric field, which complicates its application. Another technique utilizes a integrated metasurface for coupling of incident radiation into a fiber [49]. The metasurface is represented by a silicon antenna array on the surface of the $\mathrm{Si}_{3} \mathrm{~N}_{4}$ waveguide. To summarize, the devices based on hybrid plasmonic waveguides and metasurfaces are very compact, but difficult to be compatible with commercial PIC fabrication processes. Such compatibility is possessed by the design proposed in [50]: radiation is injected into two waveguides using edge-coupling forming TE01 and TE10 modes, which are subsequently combined with a $\pi / 2$ or $-\pi / 2$ phase shift. However, to obtain a vortex beam, the TE00 and TM00 modes are required to be simultaneously introduced to the edgecouplers, which also presents certain technological inconveniences.

Thus, to the best of our knowledge, an urgent task to develop a PIC for in-plane vortex beams generation, which can be manufactured using available MPW technologies remains unsolved. In this paper, we propose to use diffraction gratings for the radiation coupling into the
PIC, which makes it possible to minimize the number of directional couplers. In our approach, the vortex mode is generated in the waveguide directly from the beams incident to the waveguide gratings from standard singlemode fibers (SMF). A basic description of the proposed device is provided in the next section.

\section{Concept and operation principle}

The total view of the proposed device is presented in fig. 1. It utilizes the principle of vortex mode generation $(l= \pm 1)$ by coherent superposition in multimode waveguide on the two high-ordered TE-like modes (TE01 and TE10), one of which is phase-shifted relative to the other by $\pi / 2$ for positive topological charge and $-\pi / 2$ for a negative one. The challenging task arising in this approach is the simultaneous excitation of two high-order modes in one waveguide. A consistent solution for generating the TE01 mode is its conversion from the fundamental mode of incident radiation using the grating coupler (GC). Thus, in the proposed device, SMF placed at a properly selected tilt to the chip plane feeds the signal to the GC applied over an integrated waveguide with a width of 10 microns. The trench depth and period of GC are optimized for the efficient TE01 mode excitation.

Generating TE10 directly from a GC is a confusing technological problem, for which we propose to use a scheme based on a directional coupler (DC). At the first step, the fundamental TE00 mode is excited in the upper arm of the structure (fig. 1) provided by an optimized GC. To convert it into the TE10 mode, we use the principle of phase matching, which is implemented by selecting the widths of two adjacent waveguides so that when energy is coupled from one to the other, the fundamental mode is transformed into the desired one.

The dispersion relationship, i.e. the dependence of the effective refractive index for different modes on the waveguide width, calculated for a $500 \mathrm{~nm}$ thickness of waveguide supporting TE01 mode propagation is shown in fig. 2. Following the results obtained, the width of the waveguides required for successful conversion is respectively equal to $285.3 \mathrm{~nm}$ for TE00 propagating waveguide and $610.8 \mathrm{~nm}$ for TE10 (grey solid line).

In fig. 1, the $\mathrm{T} 1$ and $\mathrm{T} 2$ values determine the taper lengths for the TE00 and TE01 modes, respectively. They are calculated to provide transmission into narrow waveguides with a minimum modal distortion. The DC1 coupler length was determined using a Lumerical EME solver to be $15.8759 \mu \mathrm{m}$. The L1 and L4 values determine the lengths of the waveguides between the input gratings and the tapers. The length L3 is determined from the distance between the fibers and the bending radii of the waveguides R1 and R2.

Since the waveguide geometry in the process of chip fabrication, in the general case, randomly varies and statistically relates to the design parameters, the phase of the electromagnetic field for the TE00 mode at the DC's input cannot be accurately determined. Therefore, the pro- 
vision of the necessary phase shift between the TE01 and TE10 modes is provided by a phase shifter (curve over in the upper arm in fig. 1).

P. 1 in fig. 1 is the cross-section of the target waveguide in which the formed vortex mode is observed. The gap between the coupler waveguides is $280 \mathrm{~nm}$. Geometric dimensions of the specific parts of the proposed scheme are given in Table 1.

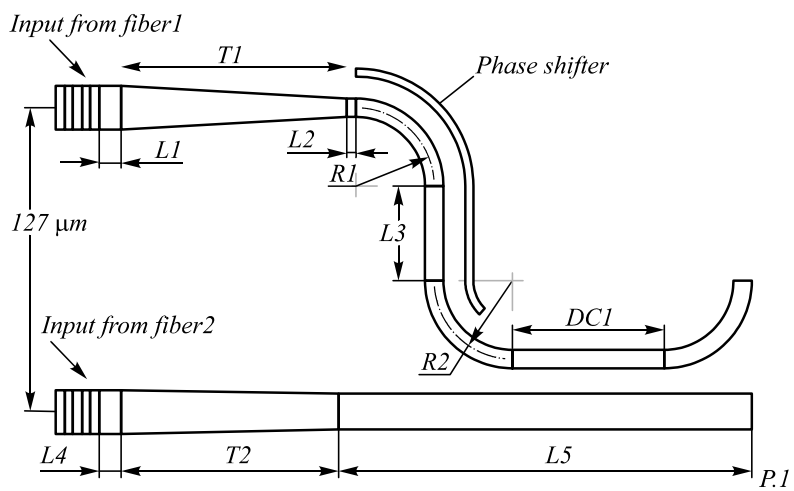

Fig. 1. Total view: L1-L5 - lengths of straight waveguides; T1$T 2$ - lengths of tapers; $R 1-R 2$ - bend radii;

DC1 - directional coupler length; P.1 - cross-section of target waveguide

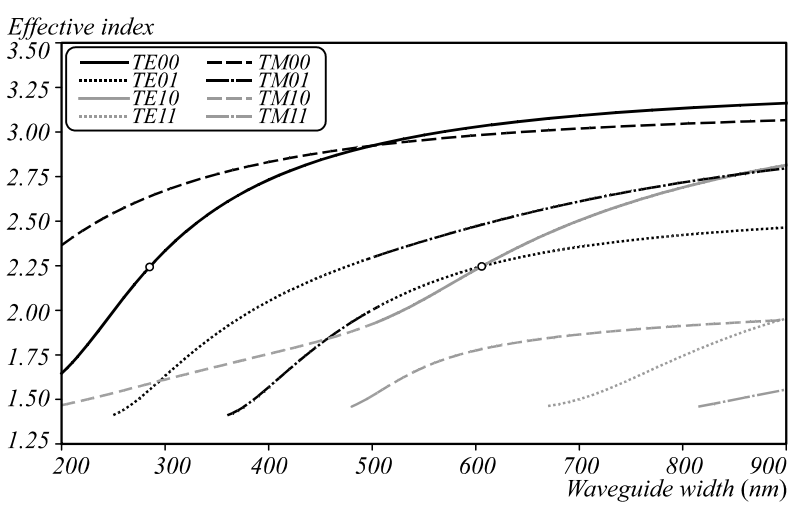

Fig. 2. Dispersion relationship

Table 1. List of geometric values from Fig. 1

\begin{tabular}{|l|c|}
\hline \multicolumn{1}{|c|}{ Dimension } & Value, $\mu \mathrm{m}$ \\
\hline L1 / L2 / L4 & 10 \\
\hline L3 & 26.27 \\
\hline L5 & 215 \\
\hline T1 & 465 \\
\hline T2 & 430 \\
\hline R1 / R2 & 50 \\
\hline DC1 & 15.87 \\
\hline
\end{tabular}

\section{Numerical simulations}

To ensure the maximum stability of the vortex field parameters in the output waveguide, it is necessary to ensure the equality of the TE01 and TE10 modes intensities in the DC's section where they will be combined, all while the intensity of all other modes is minimized.

The coupling efficiency of incident radiation into the target mode provided by GC is different for the TE00 and TE01 modes; therefore, we first optimized the period and the tilt of the feeding fiber to the chip plane normal aimed at the most efficient excitation of the TE00 mode in the integrated waveguide, which is due to the complexity of coupling to a fundamental mode in thick waveguides (more often the thickness of SOI waveguides near $200 \mathrm{~nm}$ ).

We use the Lumerical FDTD solver to simulate coupling and get a field distribution in a silicon waveguide. Then, we use elaborated scripts for modal decomposition, expansion coefficients are obtained from the equation (1):

$$
a_{\mu v}=\iint_{R^{2}} \mathbf{E}_{\mu v}^{*}(\mathbf{r}) \mathbf{E}(\mathbf{r}) d^{2} \mathbf{r},
$$

where $\mathbf{r}$ is the radius vector, $\mathbf{E}(\mathbf{r})$ is the output electric field vector, $\mathbf{E}_{\mu v}(\mathbf{r})$ is the waveguide eigenmode electric field vectors.

The simulation results are shown in fig. 3-5. The socalled relative mode purity (RMP) parameter, i.e. a ratio of target mode amplitude (TE00 in our case) to the sum of parasite modes amplitudes is presented in fig. 3 .

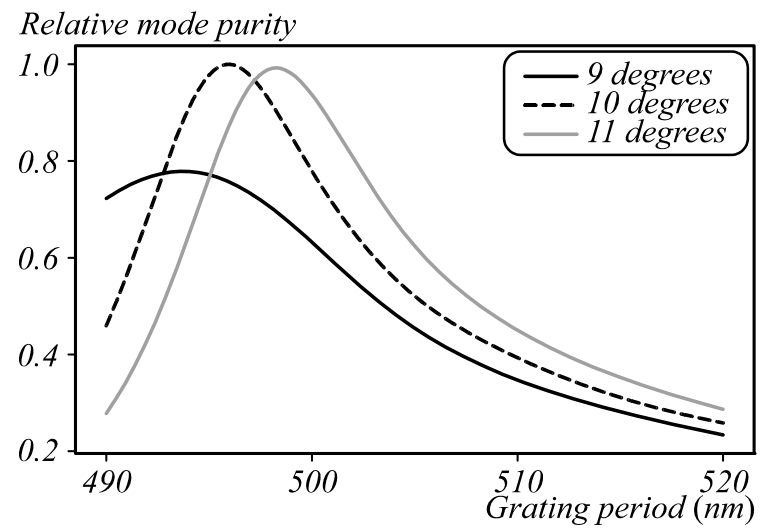

Fig. 3. Relative mode purity for TE00 excitation case

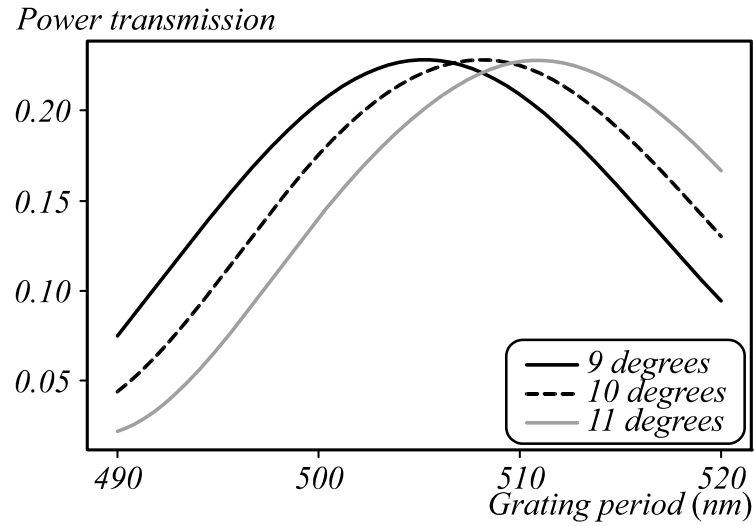

Fig. 4. Total transmission for TEO0 excitation case

Fig. 4 shows the total power transmission, in other words, the coupling efficiency of the input Gaussian beam to the waveguide's fundamental TE00 mode, while fig. 5 demonstrates RMP multiplied by amplitude transmission.

The optimal parameters are the period $497 \mathrm{~nm}$, the tilt of the fiber 10 degrees. Based on the technological feasibility, the trench depth of the grating is taken equal to $20 \%$ of the waveguide thickness. Since the simultaneous feeding of two gratings requires the arrangement of an array of fibers next to the chip, to make it technologically feasible it is necessary to ensure the same fiber tilt for both GCs. The performed 
numerical simulation showed that this parameter insignificantly affects the efficiency of TE01 mode excitation; therefore, we adopt the same tilt of 10 degrees for both fibers.

The geometry of the GC for the TE01 mode excitation is numerically optimized to ensure maximum mode purity and equal coupling efficiency for the TE00 and TE01 modes. The simulation results for a filling factor set to 0.65 are shown in fig. 6,7 .

Fig. 6 represents the period dependence of target TE01 mode and the sum of parasite modes, and fig. 7 shows the power transmission. According to the results, we chose $660 \mathrm{~nm}$ due to the relatively high mode purity and satisfying power transmission (close to the TE00 coupling power transmission).

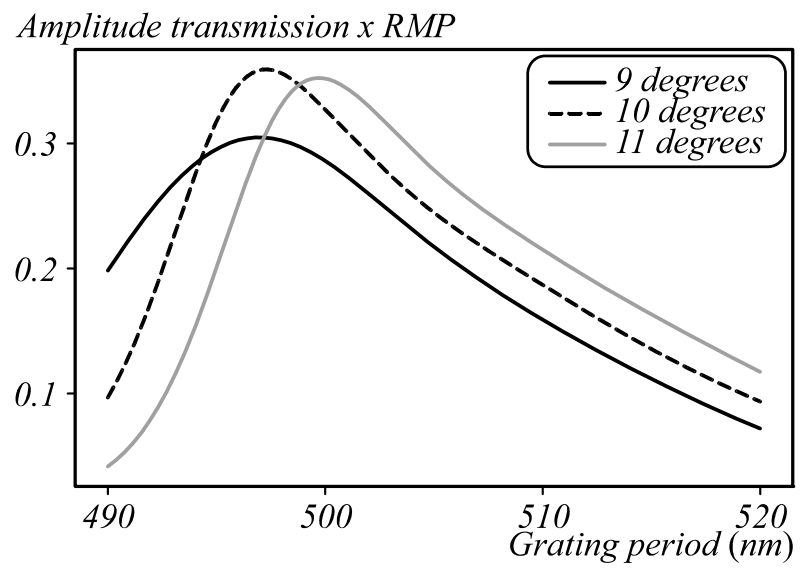

Fig. 5. Modal efficiency of TE00 excitation

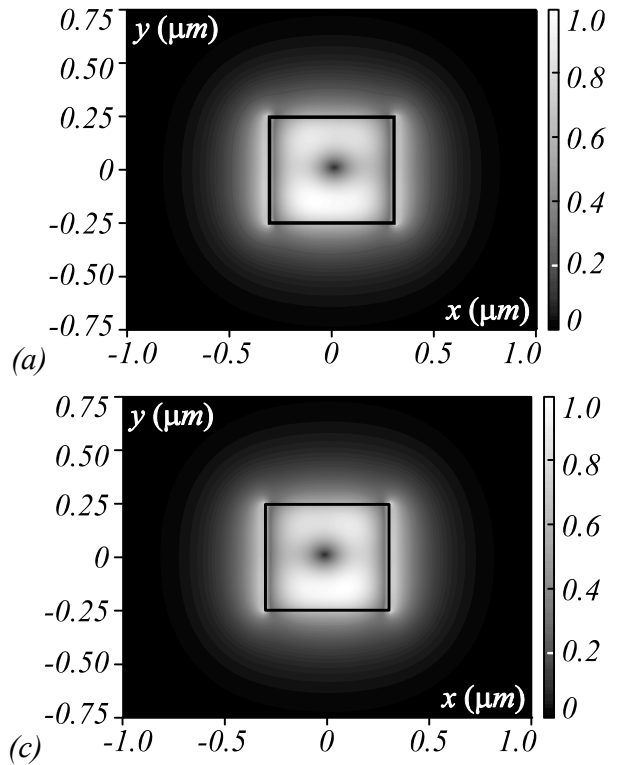

Fig. 8. Mode fields at the structure output: (a) amplitude and (b) phase for $l=+1$; c) amplitude and d) phase for $l=-1$

The simulation results of the electromagnetic field obtained at the output of the proposed PIC design after applying the required phase shift are provided in fig. 8. As we see, the amplitude distributions for both +1 and -1 OAM states have clearly distinguished zero intensity at the center of the waveguide. Also, the phase distribution demonstrates a steady phase change from $+\pi$ to $-\pi$. It is quite obvious that a vortex is generated at the output of the device.

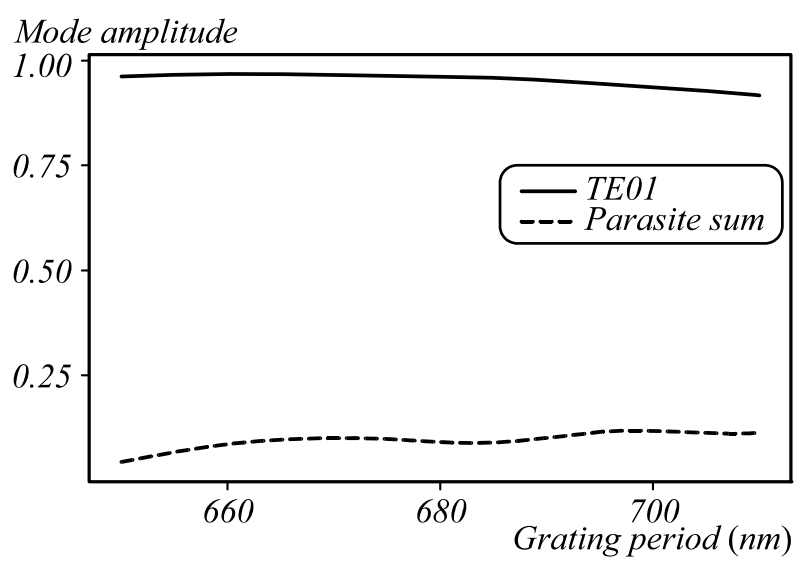

Fig. 6. Efficiency of TE01 excitation on grating period

Power transmission

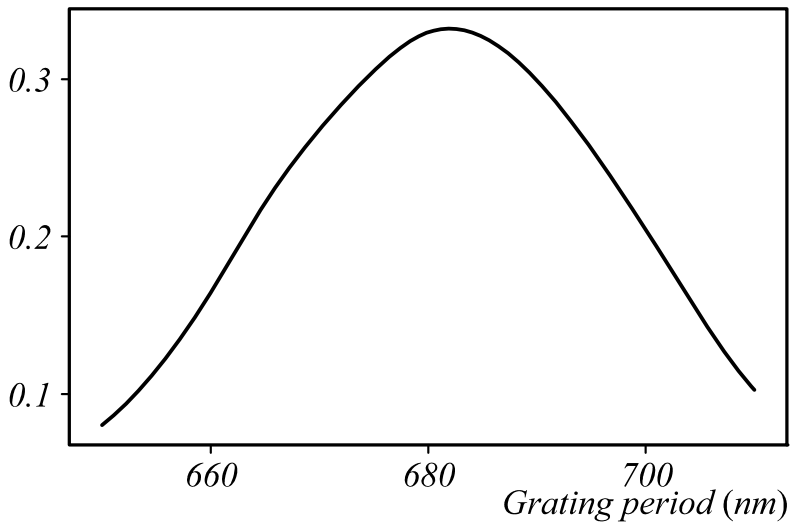

Fig. 7. Total transmission for TE01 excitation case
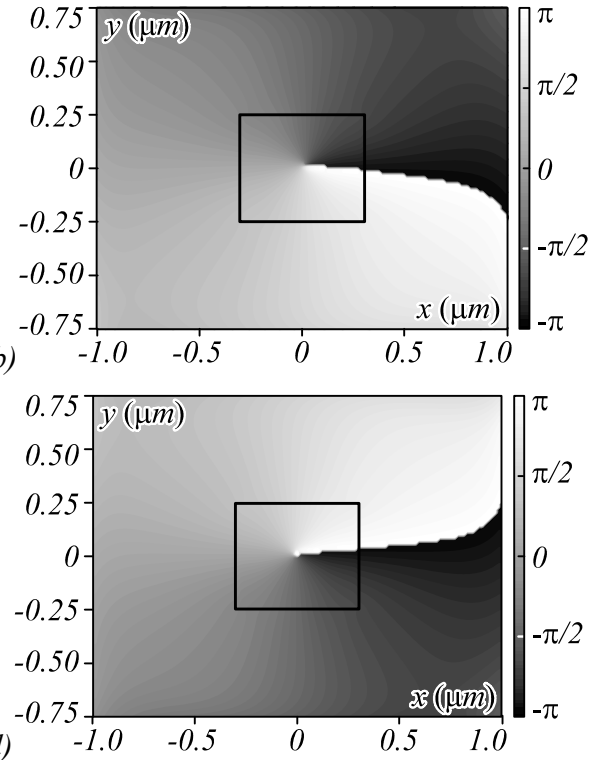

Both bandwidth purity analysis of OAM states, as well as beam power, are represented in fig. 9. It can be seen that the proposed device can be used in a wide range of wavelengths in the C-band, however, the maximums of power transmission and OAM purity do not coincide. Therefore, the actual range of applicability of the device depends on the practical requirements for the output parameters. 


\section{Conclusion}

In this paper, we propose a chip design for exciting a first-order optical vortex mode inside an integrated silicon waveguide. The optical vortex mode is combined of the TE10 and TE01 modes having a relative phase shift of $\pi / 2$ in a single waveguide. To implement this, we apply two waveguides of different widths, in which the TE00 and TE01 modes are excited from the incident radiation based on the grating coupler. The waveguides are coupled together providing a complete coupling of the TE00 mode from the first one to the TE10 mode in the second one. The geometric parameters of the elements, as well as the fiber tilt, are optimized through numerical simulation considering the options of the silicon-on-insulator fabricating technology. Moreover, the proposed method for calculating and optimizing the structure can be applied to other technological platforms that support grating couplers.

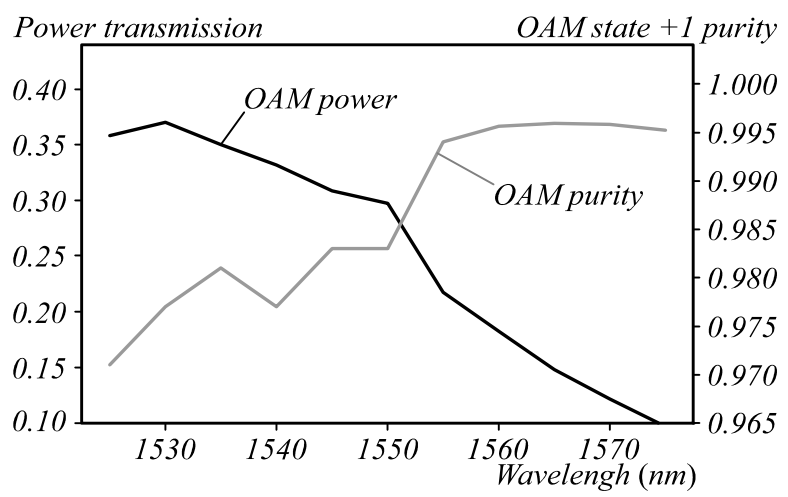

Fig. 9. OAM state purity and power transmission versus wavelength

\section{References}

[1] Bozinovic N, Yue Y, Ren Y, Tur M, Kristensen P, Huang H, Willner AE, Ramachandran S. Terabit-scale orbital angular momentum mode division multiplexing in fibers. Science 2013; 340: 1545-1548. DOI: 10.1126/science.1237861.

[2] Vasilyev VS, Kapustin AI, Skidanov RV, Podlipnov VV, Ivliev NA, Ganchevskaya SV. Experimental investigation of the stability of Bessel beams in the atmosphere. Computer Optics 2019; 43(3): 376-384. DOI: 10.18287/24126179-2019-43-3-376-384.

[3] Khonina SN, Volotovskiy SG, Kirilenko MS. A method of generating a random optical field using the Karhunen-Loeve expansion to simulate atmospheric turbulence. Computer Optics 2020; 44(1): 53-59. DOI: 10.18287/2412-6179-CO-680.

[4] Bozinovic N, et al. Terabit-scale orbital angular momentum mode division multiplexing in fibers. Science 2013; 340(6140): 1545-1548.DOI: 10.1126/science.1237861.

[5] Nicolas A, Veissier L, Giner L, Giacobino E, Maxein D, Laurat J. A quantum memory for orbital angular momentum photonic qubits. Nat Photon 2014; 8: 234-238. DOI:10.1038/nphoton.2013.355.

[6] Savelyev DA, Khonina SN. Characteristics of sharp focusing of vortex Laguerre-Gaussian beams. Computer Optics 2015; 39(5): 654-662. DOI: 10.18287/0134-2452-2015-395-654-662.

[7] Khonina SN, Savelyev DA, Kazanskiy NL. Vortex phase elements as detectors of polarization state. Opt Express 2015; 23(14): 17845-17859. DOI: 10.1364/OE.23.017845.
[8] Khonina SN, Savelyev DA, Kazanskiy NL. Analysis of polarisation states at sharp focusing. Optik 2016; 127(6): 3372-3378. DOI: 10.1016/j.ijleo.2015.12.108.

[9] Kharitonov SI, Khonina SN, Volotovskiy SG, Kazanskiy NL. Caustics of the vortex beams generated by vortex lensesand vortex axicons. J Opt Soc Am A 2020; 37(3): 476-482. DOI: 10.1364/JOSAA.382361.

[10] Khonina SN, Ustinov AV. Focusing of shifted vortex beams of arbitrary order with different polarization. Opt Commun 2018; 426: 359-365. DOI: 10.1016/j.optcom.2018.05.070.

[11] Grier DG. A revolution in optical manipulation. Nature 2003; 424(6950): 810-816. DOI: 10.1038/nature01935.

[12] Padgett M, Bowman R. Tweezers with a twist. Nat Photon 2011; 5(6): 343-348. DOI: 10.1038/nphoton.2011.81.

[13] Chapin SC, Germain V, Dufresne ER. Automated trapping, assembly, and sorting with holographic optical tweezers. Opt Express 2006; 14(26): 13095-13100. DOI: 10.1364/OE.14.013095.

[14] Gong L, et al. Optical forces of focused femtosecond laser pulses on nonlinear optical Rayleigh particles. Photonics Res 2018; 6(2): 138-143. DOI: 10.1364/PRJ.6.000138.

[15] Zhang YQ, et al. Nonlinearity-induced multiplexed optical trapping and manipulation with femtosecond vector beams. Nano Lett 2018; 18(9): 5538-5543. DOI: 10.1021/acs.nanolett.8b01929.

[16] Reddy SG, Chithrabhanu P, Vaity P, Aadhi A, Prabhakar $\mathrm{S}$, Singh RP. Non-diffracting speckles of a perfect vortex beam. J Opt 2016; 18(5): 055602.

[17] Fürhapter S, et al. Spiral phase contrast imaging in microscopy. Opt Express 2005; 13(3): 689-694. DOI: 10.1364/OPEX.13.000689.

[18] Tamburini F, et al. Overcoming the Rayleigh criterion limit with optical vortices. Phys Rev Lett 2006; 97(16): 163903. DOI: 10.1103/PhysRevLett.97.163903.

[19] Jesacher A, Ritsch-Marte M, Piestun R. Three-dimensional information from two-dimensional scans: A scanning microscope with postacquisition refocusing capability. Optica 2015; 2(3): 210-213. DOI: 10.1364/OPTICA.2.000210.

[20] Willig KI, et al. STED microscopy reveals that synaptotagmin remains clustered after synaptic vesicle exocytosis. Nature 2006; 440(7086): 935-939. DOI: 10.1038/nature04592.

[21] Zhao Y, et al. Chirality detection of enantiomers using twisted optical metamaterials. Nat Commun 2017; 8(1): 14180. DOI: $10.1038 /$ ncomms 14180.

[22] Xie G, Song H, Zhao Z, et al. Using a complex optical orbitalangular-momentum spectrum to measure object parameters. Opt Lett 2017; 42: 4482-4485. DOI: 10.1364/OL.42.004482.

[23] Xie ZW, et al. On-chip spin-controlled orbital angular momentum directional coupling. J Phys D Appl Phys 2017; 51(1): 014002.

[24] Lavery $M$, et al. Detection of a spinning object using light's orbital angular momentum. Science 2013; 341: 537540. DOI: $10.1126 /$ science. 1239936.

[25] Cvijetic N, et al. Detecting lateral motion using light's orbital angular momentum. Sci Rep 2015; 5(1): 15422. DOI: 10.1038/srep15422.

[26] Zhang H, Mao B, Han Y, Wang Z, Yue Y, Liu Y. Generation of orbital angular momentum modes using fiber systems. Appl Sci 2019; 9(5): 1033. DOI: 10.3390/app9051033.

[27] Khonina SN, Kazanskiy NL, Soifer VA. Optical vortices in a fiber: Mode division multiplexing and multimode selfimaging. In Book: Recent Progress in Optical Fiber Research. Chap 15. London: IntechOpen; 2012: 327-352. DOI: $10.5772 / 28067$.

[28] Courtial J, Padgett MJ. Performance of a cylindrical lens mode converter for producing Laguerre-Gaussian laser 
modes. Opt Commun 1999; 159(1-3): 13-18. DOI: 10.1016/S0030-4018(98)00599-9.

[29] Beijersbergen MW, Coerwinkel RPC, Kristensenl M, Woerdman JP. Helical-wavefront laser beams produced with a spiral phaseplate. Opt Commun 1994; 112(5-6): 321-327. DOI: 10.1016/0030-4018(94)90638-6.

[30] Uchida M, Tonomura A. Generation of electron beams carrying orbital angular momentum. Nature 2010; 464(7289), 737-739. DOI: 10.1038 /nature 08904 .

[31] Heckenberg NR, McDuff R, Smith CP, White AG. Generation of optical phase singularities by computergenerated holograms. Opt Lett 1992; 17(3): 221-223. DOI: 10.1364/OL.17.000221.

[32] Zhe Z, Wang J, Willner AE. Metamaterials-based broadband generation of orbital angular momentum carrying vector beams. Opt Lett 2013; 38(6): 932-934. DOI: 10.1364/OL.38.000932.

[33] Marrucci L, Karimi E, Slussarenko S, Piccirillo B, Santamato E, Nagali E, Sciarrino F. Spin-to-orbital conversion of the angular momentum of light and its classical and quantum applications. J Opt 2011; 13(6): 064001.

[34] Wu H, Gao SC, Huang BS, Feng YH, Huang XC, Liu WP, Li ZH. All-fiber second-order optical vortex generation based on strong modulated long-period grating in a fourmode fiber. Opt Lett 2017; 42(24): 5210-5213. DOI: 10.1364/OL.42.005210.

[35] Ma X, Liu CH, Chang G, Galvanauskas A. Angularmomentum coupled optical waves in chirally-coupled-core fibers. Opt Express 2011; 19(27): 26515-26528. DOI: 10.1364/OE.19.026515.

[36] Zeng XL, Lin Y, Feng LP, Wu SH, Yang C, Li W, Tong WJ, Wu J. All-fiber orbital angular momentum mode multiplexer based on a mode-selective photonic lantern and a mode polarization controller. Opt Lett 2018; 43(19): 47794782. DOI: $10.1364 /$ OL.43.004779.

[37] Wang X, Nie Z, Liang Y, Wang J, Li T, Jia B. Recent advances on optical vortex generation. Nanophotonics 2018 7(9): 1533-1556. DOI: 10.1515/nanoph-2018-0072.

[38] Li R, Feng X, Zhang D, Cui K, Liu F, Huang Y. Radially polarized orbital angular momentum beam emitter based on shallow-ridge silicon microring cavity. IEEE Photon J 2014; 6(3): 1-10. DOI: 10.1109/JPHOT.2014.2321757.

[39] Wang Y, et al. Integrated photonic emitter with a wide switching range of orbital angular momentum modes. Sci Rep 2016; 6: 22512. DOI: 10.1038/srep22512.
[40] Scaffardi M, et al. $3 \times 3$ optical switch by exploiting vortex beam emitters based on silicon microrings with superimposed gratings. Opt Lett 2017; 42(19): 3749-3752. DOI: 10.1364/OL.42.003749.

[41] Xie Z, Lei T, Li F, et al. Ultra-broadband on-chip twisted light emitter for optical communications. Light Sci Appl 2018; 7: 18001. DOI: 10.1038/lsa.2018.1.

[42] Li H, Phillips DB, Wang X, et al. Orbital angular momentum vertical-cavity surface-emitting lasers. Optica 2015; 2(6): 547-552. DOI: 10.1364/OPTICA.2.000547.

[43] Paul S, et al. Wavelength-selective orbital-angularmomentum beam generation using MEMS tunable FabryPerot filter. Opt Lett 2016; 41(14): 3249-3252. DOI: 10.1364/OL.41.003249.

[44] Lyubopytov VS, Kutluyarov RV, Bagmanov VK, Neumann N, Sultanov AK. Modeling and optimization of vortex modes propagation in rectangular dielectric waveguides. IEEE Photon J 2020; 12(1): 1-17. DOI: 10.1109/JPHOT.2019.2958273.

[45] Zheng S, Wang J. On-chip orbital angular momentum modes generator and (de)multiplexer based on trench silicon waveguides. Opt Express 2017; 25(15): 18492-18501. DOI: 10.1364/OE.25.018492.

[46] Liang Y, Wu H, Huang B, Huang XG. Light beams with selective angular momentum generated by hybrid plasmonic waveguides. Nanoscale 2014; 6: 12360-12365. DOI: $10.1039 / C 4 N R 03606$ A.

[47] Liang Y, Zhang F, Gu J, Huang XG, Liu S. Integratable quarter-wave plates enable one-way angular momentum conversion. Sci Rep 2016; 6: 24959. DOI: $10.1038 /$ srep24959.

[48] Ni FC, Xie ZT, Hu X, Jia C, Huang XG. Selective angular momentum generator based on a graphene hybrid plasmonic waveguide. J Lightw Technol 2019; 37(21): 5486-5492.

[49] Meng Y, Liu Z, et al. Versatile on-chip light coupling and (de)multiplexing from arbitrary polarizations to controlled waveguide modes using an integrated dielectric metasurface. Photonics Res 2020; 8(4): 564-576. DOI: 10.1364/PRJ.384449.

[50] Zhang D, Feng X, Cui K, Liu F, Huang Y. Generating inplane optical orbital angular momentum beams with silicon waveguides. IEEE Photon J 2013; 5(2): 2201206. DOI: 10.1109/JPHOT.2013.2256888.

\section{Authors' information}

Ruslan Vladimirovich Kutluyarov (b. 1986) graduated with honors (2008) from Ufa State Aviation Technical University (USATU), majoring in Multichannel Telecommunication Systems. He has defended Candidate of Sciences thesis in 2013. Currently he works as the senior researcher and assistant professor at the Telecommunication Systems department in USATU. Current research interests include integrated photonics, nonlinear optics, fiber optics, microwave photonics, quantum communications. E-mail: kutluyarov.rv@ugatu.su .

Denis Marsovich Fatkhiev (b. 1997) graduated with honors (2019) from Ufa State Aviation Technical University (USATU), majoring in Infocommunication Technologies and Communication Systems. Currently he works as the research engineer at the USATU, Telecommunication Systems department. Current research interests are integrated photonics, nonlinear optics, optical communications. E-mail: fatkhiev.dm@ugatu.su.

Ivan Vasilevich Stepanov, (b. 1998) graduated with honors (2020) from Ufa State Aviation Technical University (USATU), majoring in Infocommunication Technologies and Special Communication Systems. Currently he is a PhD student at the USATU and works as the engineer at the Telecommunication Systems department. Current research interests are integrated photonics, optical communications. E-mail: ivan-stepanov-2013@list.ru. 
Elizaveta Pavlovna Grakhova (b. 1990) graduated with honors (2012) from Ufa State Aviation Technical University, majoring in Radiocommunication, Radiobroadcasting and Television. She has defended Candidate of Sciences thesis in 2016. Currently she works as assistant professor and senior researcher at the USATU, Telecommunication Systems department. Current research interests include signal processing, antenna technology, microwave photonics.

E-mail: grakhova.ep@ugatu.su.

Vladimir Sergeevich Lyubopytov (b. 1986) graduated with honors (2008) from Ufa State Aviation Technical University, majoring in Multichannel Telecommunication Systems. He has defended Candidate of Sciences thesis in 2013. Currently he works as a senior research scientist at the Center for Photonics and Quantum Materials of the Skolkovo Institute of Science and Technology. Current research interests include high-speed optical communications, integrated photonics, digital and optical signal processing. E-mail: lyubopytov.v.ru@ieee.org.

Albert Khanovich Sultanov (b. 1950) graduated with honors (1973) from the Novosibirsk Electrotechnical Institute of Telecommunications (at present Siberia State University of Telecommunications and Informatics), majoring in Multichannel Electrocommunication. He received his Doctor in Technical Sciences degree (1996) from Ufa State Aviation Technical University (USATU). Currently he is a Head of the Telecommunication Systems department at the USATU. He is a SPIE member, Academic of Telecommunication Academy. Current research interests include telecommunications, fiber optics, integrated photonics, aerospace systems, microsatellite communications. E-mail: tks@ugatu.ac.ru.

Code of State Categories Scientific and Technical Information (in Russian - GRNTI)): 29.33.39. Received May 30, 2016. The final version - January 11, 2020. 